Volodymir G. Zinkovsky (Opole)

Olga V. Zhuk (Opole)

Michae TEODORCZyK (Opole)

NATALIA KARPINCHIK (Odessa)

\title{
FORMALISATION AND METHODS OF ANALYSIS OF THE FAST XENOBIOTIC MASS TRANSFER IN THE BODY
}

Abstract. A novel discrimination and regression method for a quantitative determination of the relative efficiency of "fast" distribution processes of xenobiotics is discussed. An integral model-independent method for estimation of equilibrium tissue-to-plasma partition ratios is proposed.

1. Introduction. Modelling of xenobiotic distribution assumes that a body consists of central and several peripheral compartments representing blood and different organs and tissues $[4,8]$. Exchange of xenobiotic between the central and peripheral compartments occurs by diffusion mass transfer. In some compartments, metabolic and/or excretion elimination of substances takes place. Mixing inside compartments eliminates xenobiotic concentration gradients [11], therefore the rates of mass transfer between central and peripheral compartments are equal to the product of xenobiotic contents in each of these compartments and some first order rate constants [11, 12]. The values of mass transfer rate constants are determined by the product of equally accessible phase partition surfaces (between central and peripheral compartments [12]) and corresponding diffusion rate constants [6]. It should be noted that neither of these parameters could be determined during an in vivo experiment. In conventional pharmacokinetic studies, experimental data are a series of xenobiotic concentrations in blood $\left(C_{1 t}\right)$ and other organs and tissues $\left(C_{i t}\right.$, as we shall limit our analysis to the compartment (i) only) in various time intervals following its administration. Therefore,

2000 Mathematics Subject Classification: 92C45.

Key words and phrases: integral of xenobiotic concentration, balance equation of xenobiotic mass transfer, regression analysis, discrimination method. 
the mass balance equation for the compartment $i$ at time $t$ after xenobiotic administration is written as

$$
V_{i}\left(\frac{d C_{i t}}{d t}\right)=V_{1} \cdot k_{1 i} \cdot C_{1 t}-V_{i} \cdot C_{i t} \cdot \sum k_{i},
$$

where: $V_{i}$ and $V_{1}$ are the volumes of the central compartment (1) and the compartment $(i)$; respectively, $\sum k_{i}=k_{i 1}+k_{i 0}$ is the sum of the rate constants of mass transfer from the compartment $(i)$ to compartment (1) and of its elimination from the compartment; $k_{1 i}$ is the rate constant of diffusion from the central compartment to the compartment $(i)$.

2. Methods for determination of xenobiotic distribution constants, based on direct measurements of $C_{1 t}$ and $C_{i t}$. We assume identical and equally accessible partition surfaces for the diffusion from (1) to $(i)$ and from $(i)$ to (1). In conditions of prolonged xenobiotic administration at a constant rate (usually intravenously), steady-state concentrations are achieved in blood and other compartments. In such conditions, the value of $\frac{d C_{i t}}{d t}$ approaches zero and therefore

$$
\frac{C_{i t}}{C_{1 t}} \approx \frac{V_{1} k_{1 i}}{V_{i} \sum k_{i}}=K_{p}
$$

where $K_{p}$ is the constant of xenobiotic distribution between (1) and (i). The value of $K_{p}$ when $\sum k_{i}=k_{i 1}$ and $k_{i 0}=0$ is equal to the ratio of the rate constants of its diffusion into (1) and $(i)$, i.e. corresponds to the value of the actual thermodynamic constant of xenobiotic distribution between the compartments.

Following a single pulse administration of a xenobiotic dose into any compartment other than $(i)$, its concentration in the compartment $(i)$ increases with the duration of the experiment and reaches a maximum value at $t=T_{\max , i}$, which is variable between different compartments. The derivative of the concentration becomes zero and this allows interpreting experimental data at $t=T_{\max , i}$ in accordance with equation (2). At $t<T_{\max }$, the value of $C_{i t} / C_{1 t}$ is less than $K_{p}$; at $t=T_{\max }$ it is equal to $K_{p}$, while at $t>T_{\max , i}$ it is bigger than $K_{p}$ and asymptotically approaches its real value. This makes it possible to use the graph in coordinates $\left[C_{i t} / C_{1 t}, t\right]$ to determine the values of $K_{p}$ for the xenobiotic distribution between the central and peripheral compartments.

\section{Methods for determination of constants of xenobiotic distri-} bution between blood and tissues based on integral parameters. In modern model-independent pharmacokinetic analysis, integrals of drug concentrations in blood and tissues are presented as "areas under pharma- 
cokinetic curves" (AUC) mostly calculated using the trapezoidal rule, for example $\int_{t_{1}}^{t_{2}} C_{i t} d t \approx A U C_{i}^{\left(t_{1}-t_{2}\right)}[2,9]$.

Integrating equation (1) from zero to infinity and from zero to $t$, assuming that for $t=0, C_{i t}=0$ (i.e. integration constants are equal to zero) we obtain:

$$
\left\{\begin{aligned}
\frac{\int_{0}^{\infty} C_{i t} d t}{\int_{0}^{\infty} C_{1 t} d t} & =\frac{V_{1} \cdot k_{1 i}}{V_{i} \sum k_{i}}=K_{p} \approx \frac{A U C_{i}^{(0-\infty)}}{A U C_{1}^{(0-\infty)}} \\
\frac{\int_{0}^{t} C_{i t} d t}{\int_{0}^{t} C_{1 t} d t} & =\frac{V_{1} \cdot k_{1 i}}{V_{i} \sum k_{i}}-\frac{C_{i t}}{\sum k_{i} \int_{0}^{t} C_{1 t} d t} \approx K_{p}-\frac{C_{i t}}{\sum k_{i} A U C_{1}^{(0-t)}} \\
& \approx \frac{A U C_{i}^{(0-t)}}{A U C_{1}^{(0-t)}}
\end{aligned}\right.
$$

The first equation of the system (3) is the basis for determining $K_{p}$ in modern pharmacokinetics $[1,4,5]$. We used anamorphosis of the second equation of the system (3) for graphic analysis of experimental data in coordinates $\left[A U C_{i}^{(0-t)}\right],\left[A U C_{1}^{(0-t)}\right]$ and $\left[A U C_{i}^{(0-t)} / A U C_{1}^{(0-t)}, t\right]$. In addition, a regression method of analysis has been proposed to determine $K_{p}$ and $\sum k_{i}[12,13]$.

4. Fast processes of xenobiotic distribution in the body. To provide experimental examples of xenobiotic distribution in the present study, we use our previously reported data on pharmacokinetics of the following radioactively labeled substances: $\left[{ }^{3} \mathrm{H}\right]$-tilorone, $\left[{ }^{14} \mathrm{C}\right]$-phenazepam and $\left[{ }^{14} \mathrm{C}\right]$ thiobarbiturates $[7,13]$.

If the xenobiotic mass transfer kinetics between blood and a tissue corresponds to the mass balance equation (1), then the ratio of blood and tissue concentration integrals is described by the characteristic linear equation of the regression analysis:

$$
\frac{\int_{0}^{t} C_{i t} d t}{C_{i t}}=K_{p} \frac{\int_{0}^{t} C_{1 t} d t}{C_{i t}}-\left(\sum k_{i}\right)^{-1} .
$$

However, we have observed full correspondence with this prediction only in the case of the distribution of $\left[{ }^{14} \mathrm{C}\right]$-thiobarbiturates in all tissues studied except for the liver (Fig. 1). Analyzing experimental data using equation (4), we revealed substantial deviations from linearity in:

(a) all tissues following intravenous administration of $\left[{ }^{3} \mathrm{H}\right]$-tilorone and in liver and lungs after its oral administration;

(b) in brain and liver after intraperitoneal administration of $\left[{ }^{14} \mathrm{C}\right]$-phenazepam;

(c) in liver after intragastrical administration of $\left[{ }^{14} \mathrm{C}\right]$-thiobarbiturates. To explain such observations, we suggested that in addition to the reversible 


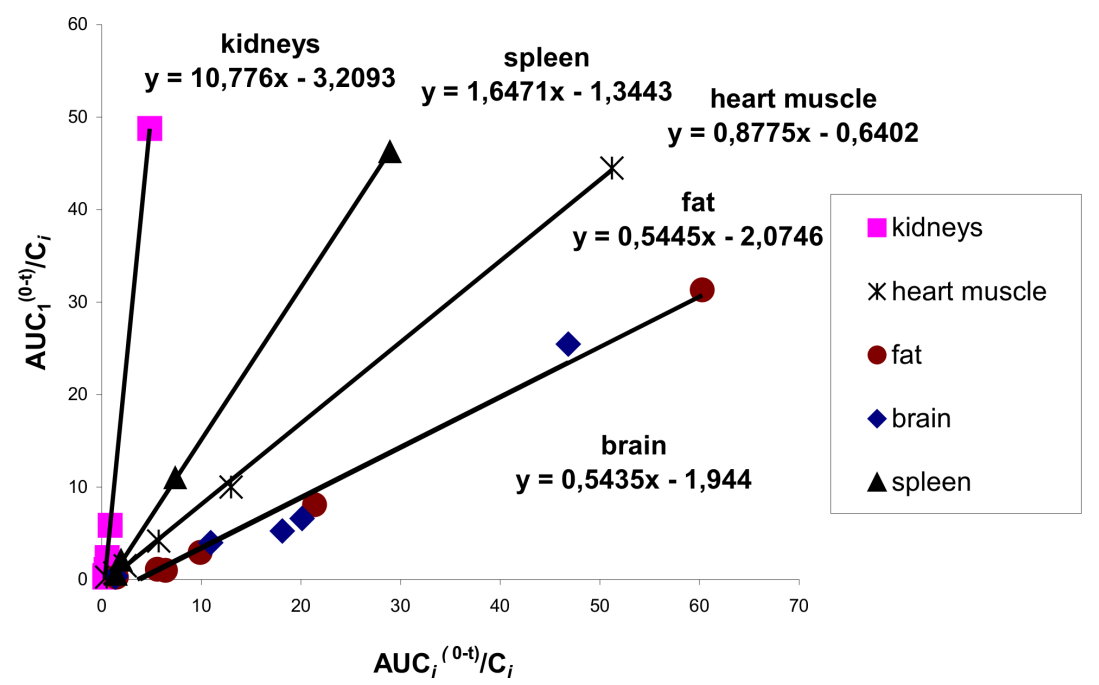

Fig. 1. The ratio between the areas under curves of the total radioactivity and tissue concentrations of the total radioactivity $\left(A U C_{i}^{(0-t)} / C_{i t}\right)$ and the ratio between the areas under curves of total radioactivity in blood and tissue concentrations of the total radioactivity $\left(A U C_{1}^{(0-t)} / C_{i t}\right)$ after intragastrical administration of $\left[{ }^{14} \mathrm{C}\right]$-thiobarbiturates into mice at a dose of $50 \mathrm{mg} / \mathrm{kg}$.

first order processes described by equation (1), there exist fast xenobiotic distributions into peripheral compartments. In the simplest case, the characteristic time of the fast process equals the reciprocal of the rate constant in the linear kinetic scheme. The characteristic time of the fast process is at least an order of magnitude less than: (a) the shortest interval of xenobiotic concentration sampling and (b) characteristic times of the processes analyzed $\left(k_{1 i}^{-1}\right.$ and $\left.k_{i}^{-1}\right)$. There is a mathematical approach to the approximate description of the fast processes according to Tikhonov's theorem [10] where the process rapidly achieving steady state in the differential equation can be modeled by an algebraic expression (in other words we assume that the process has reached its steady state). Thus integrating equation (1) from zero to $t$ and to infinity, we obtain:

$$
\left\{\begin{array}{l}
V_{i} C_{i t}=V_{1} k_{1 i} \int_{0}^{t} C_{1 t} d t-V_{i} \sum k_{i} \int_{0}^{t} C_{i t} d t+J, \\
V_{i} \sum k_{i} \int_{0}^{\infty} C_{i t} d t=V_{1} k_{1 i} \int_{0}^{\infty} C_{1 t} d t+J
\end{array}\right.
$$

where $J$ is the integration constant equal to the steady state value of the fast process, that is, to $V_{i} C_{i t}$ as $t \rightarrow 0\left(J=V_{i} C_{i 0}\right)$. 
5. Discrimination method for determination of the fast processes of xenobiotic distribution between blood and tissues. We propose a discrimination method based on the transformation of the first equation of the system (5) into:

$$
\frac{\int_{0}^{t} C_{i t} d t}{\int_{0}^{t} C_{1 t} d t}=K_{p}-\frac{C_{i t}-C_{i 0}}{\sum k_{i} \int_{0}^{t} C_{i t} d t} .
$$

Plotting the ratio of the integral of xenobiotic concentration in the tissue to the integral of its concentration in blood in coordinates

$$
\left[\int_{0}^{t} C_{i t} d t / \int_{0}^{t} C_{1 t} d t ; t\right]
$$

one can observe the following outcomes:

(a) if fast processes of xenobiotic distribution are absent, i.e. $C_{i 0}=0$, then this ratio monotonically increases with time from zero to $K_{p}$ (Fig. 2A, all tissues except for liver);

(b) if fast processes are dominant $\left(C_{i t}<C_{i 0}\right.$ at any value of $\left.t\right)$, then the ratio monotonically decreases with time approaching the value $K_{p}+C_{0 i} /\left(\sum k_{i} \int_{0}^{\infty} C_{1 t} d t\right)$ (Fig. 2A, liver; 2B);

(c) if fast processes are present but not dominant, the ratio of integrals fluctuates: it decreases initially and subsequently slowly increases approaching the value $K_{p}+C_{0 i} /\left(\sum k_{i} \int_{0}^{\infty} C_{1 t} d t\right)$ (Fig. $2 \mathrm{C}$ ).

(A)

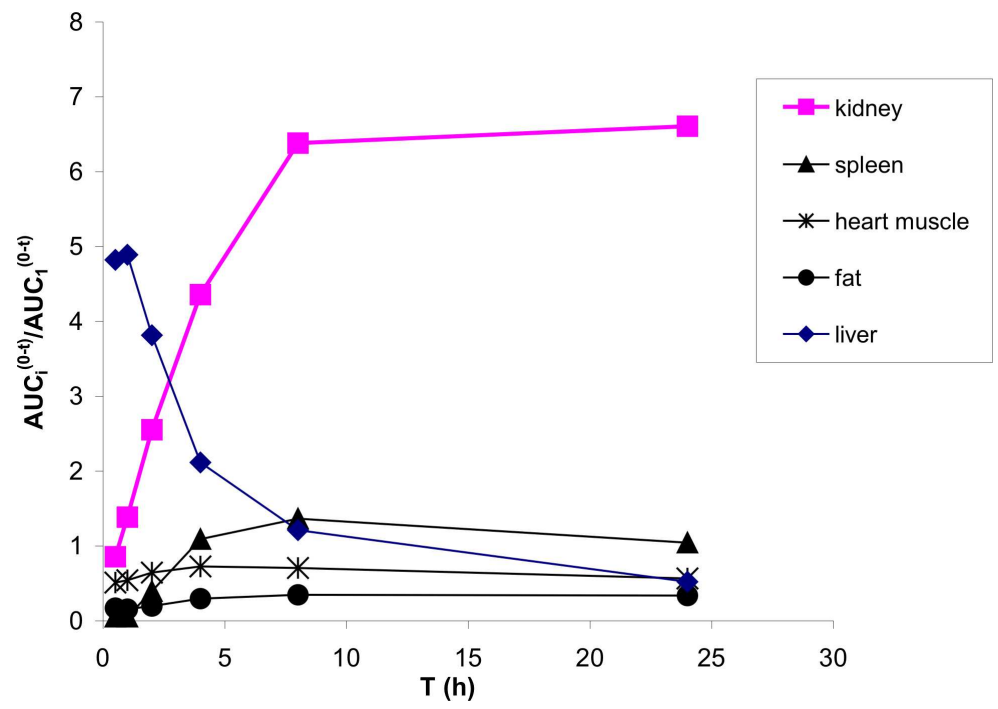


(B)

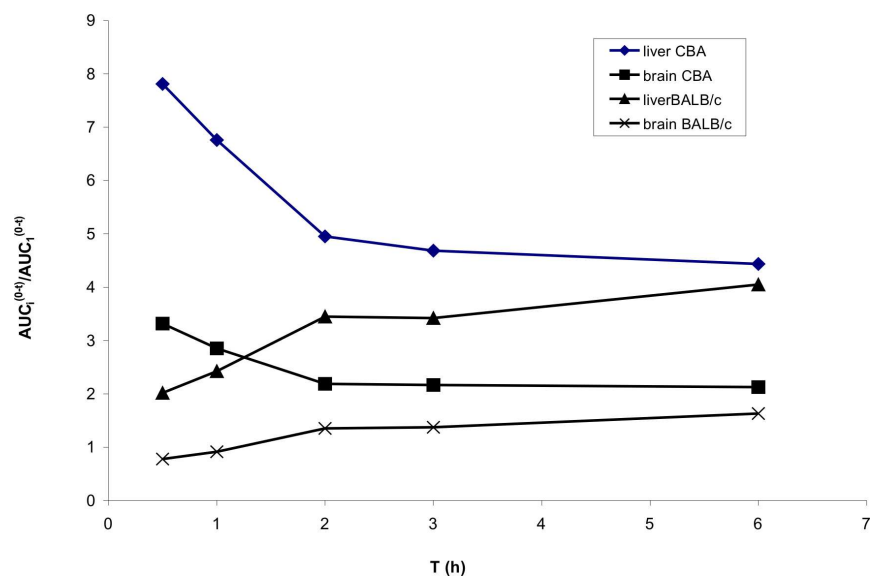

intravenous administration

(C)
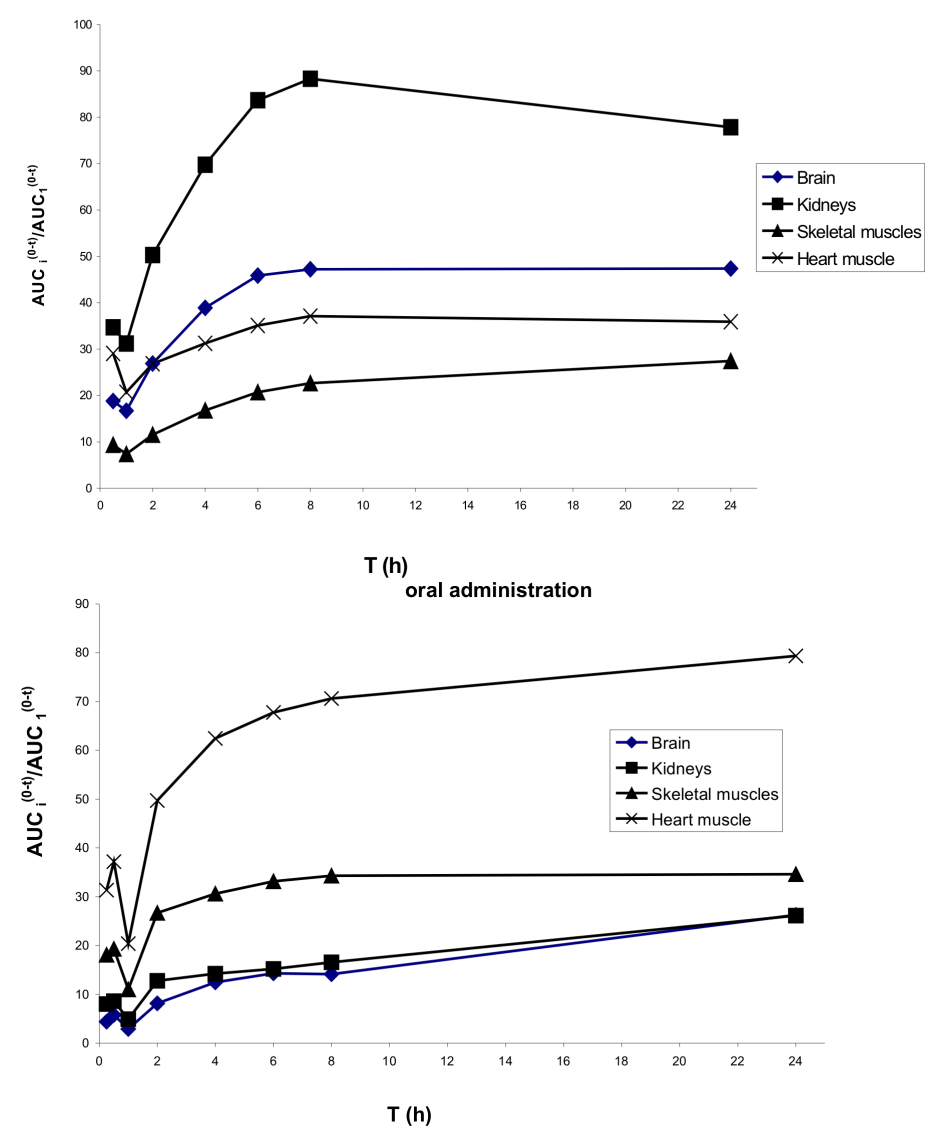

Fig. 2. $\left(A U C_{i}^{(0-t)} / A U C_{1}^{(0-t)}\right)$ as a function of time: A: after intragastrical administration of $\left[{ }^{14} \mathrm{C}\right]$-thiobarbiturates into mice at a dose of $50 \mathrm{mg} / \mathrm{kg}$; B: after intragastrical administration of $\left[{ }^{14} \mathrm{C}\right]$-phenazepam into CBA and BALB/c mice at a dose of $14 \mathrm{mg} / \mathrm{kg}$; C: after intravenous and oral administration of $\left[{ }^{3} \mathrm{H}\right]$-amixin into mice at a dose of $50 \mathrm{mg} / \mathrm{kg}$. 
6. Regression methods for determination of fast processes of xenobiotic distribution between blood and tissues. To determine the values of $K_{p}$ and $\sum k_{i}$ in the presence of fast distribution processes, it is necessary to subtract the first equation of the system (5) from the second one and regroup the resulting expression into a linear trinomial:

$$
\left(\int_{0}^{\infty} C_{i t} d t-\int_{0}^{t} C_{i t} d t\right) / C_{i t}=K_{p}\left(\int_{0}^{\infty} C_{1 t} d t-\int_{0}^{t} C_{1 t} d t\right) / C_{i t}+\frac{1}{\sum k_{i}}
$$

Differences of concentration integrals can be replaced by inverse integrals (from $+\infty$ to $t$ ). The use of inverse integrals extends the capacity of the method. If the study encompasses only ascending part of, rather than the whole, kinetic curve (from zero to $T$, where $T$ is the terminal experimental interval) and determination of either $\left(\int_{0}^{\infty} C_{i t} d t\right.$ and/or $\left.\int_{0}^{\infty} C_{1 t} d t\right)$ is not feasible, then instead of inverse integrals of the concentration it is possible to use integrals with variable lower bound $t(t<T)$. Then equation $(6)$ is transformed into

$$
\left(\int_{t}^{T} C_{i t} d t\right) /\left(C_{i t}-C_{i T}\right)=K_{p}\left(\int_{t}^{T} C_{1 t} d t\right) /\left(C_{i t}-C_{i T}\right)+\frac{1}{\sum k_{i}},
$$

where $C_{i T}$ is the xenobiotic concentration in the tissue at time $T$. An example of application of equation (8) for calculation of $K_{p}$ values of $\left[{ }^{3} \mathrm{H}\right]$-tilorone in mice is given in Fig. 3 .

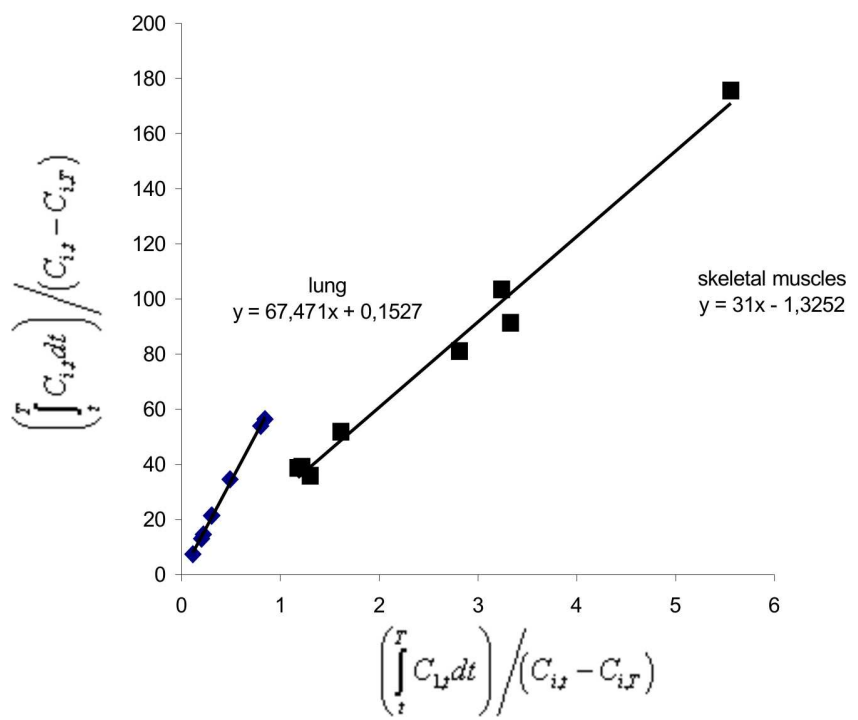

Fig. 3. Graphic interpretation of the equation (8) in coordinates $\left[\int_{t}^{T} C_{i t} d t /\left(C_{i t}-C_{i T}\right)\right.$, $\left.\int_{t}^{T} C_{1 t} d t /\left(C_{i t}-C_{i T}\right)\right]$ for the experimental data on distribution of $\left[{ }^{3} \mathrm{H}\right]$-tilorone in mice 
In the general case, for any two time intervals $\left(t_{1}\right.$ and $\left.t_{2}>t_{1}\right)$ we can obtain

$$
\left(\int_{t_{1}}^{t_{2}} C_{i t} d t\right) /\left(C_{i t_{1}}-C_{i t_{2}}\right)=K_{p}\left(\int_{t_{1}}^{t_{2}} C_{1 t} d t\right) /\left(C_{i t_{1}}-C_{i t_{2}}\right)+\frac{1}{\sum k_{i}} .
$$

Regression determination of the xenobiotic quantity that enters a unit of volume of the compartment $(i)$ as a result of the fast distribution process $\left(C_{i 0}\right)$ and the sum of its mass transfers $\left(q_{i}=\sum k_{i} \int_{0}^{\infty} C_{i t} d t\right)$ can be performed after sequential substitution and transformation of equation (7) into:

$$
\left\{\begin{array}{l}
\frac{C_{i t}}{F_{i t}-F_{1 t}}=\frac{C_{i 0}\left(1-F_{1 t}\right)}{F_{i t}-F_{1 t}}+q_{i} \\
F_{i t}=\int_{0}^{t} C_{i t} d t / \int_{0}^{\infty} C_{i t} d t \\
F_{1 t}=\int_{0}^{t} C_{1 t} d t / \int_{0}^{\infty} C_{1 t} d t
\end{array}\right.
$$

The contribution (relative efficiency) of the fast processes to the sum of all xenobiotic mass transfers into compartment $(i)$ can be expressed as $C_{i 0} / q_{i}$, whereas the absolute xenobiotic availability $F$ in the tissue is given by

$$
F=q_{i} / d
$$

where $d$ is the xenobiotic dose (mass of xenobiotic/mass of the animal).

Applying equation (10) to the experimental data of $\left[{ }^{14} \mathrm{C}\right]$-phenazepam distribution in BALB/c mice, we observed that for the liver and the brain compartment

$$
q_{i} \approx C_{i},
$$

i.e. the drug distributes into these tissues almost exclusively by the fast processes before the first sampling $30 \mathrm{~min}$ after its administration.

If the assumption of equation (2) holds true, then the distribution of phenazepam between blood, liver and brain is characterized by $K_{p} \rightarrow 0$, since $k_{l i} \rightarrow 0$. Thus the system of equations (5) is simplified as follows:

$$
C_{i t}=C_{i 0}-\sum k_{i} \int_{0}^{t} C_{i t} d t, \quad C_{i 0}=\sum k_{i} \int_{0}^{\infty} C_{i t} d t .
$$

So, the plot of experimental data in coordinates $\left[C_{i t}, A U C_{i}^{(0-t)}\right]$ should be linear and intersect the $Y$ - and $X$-axes at points $C_{i 0}$ and $C_{i 0} / \sum k_{i}$ respectively. As can be seen in Fig. 4, the validity of equation (12) is confirmed. 


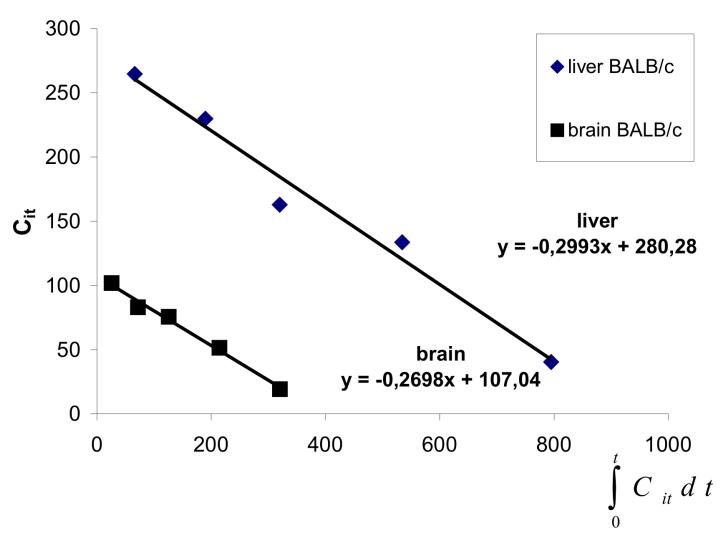

Fig. 4. Graphic interpretation of the equation (12) for the experimental data on distribution of $\left[{ }^{14} \mathrm{C}\right]$-phenazepam distribution in $\mathrm{BALB} / \mathrm{c}$ mice

7. Conclusion. There could be several reasons for fast processes of xenobiotic distribution into tissues, discussed in the paper. We shall name the most obvious ones:

(a) Hepatic and pulmonary first-pass uptake of the drug following its oral or intraperitoneal administration may precede its entrance into the arterial blood stream and subsequent distribution from blood to other tissues. In conditions of intravenous injection, first-pass effect is noted mainly in lungs.

(b) The ratio of concentrations of free and protein bound xenobiotic may fluctuate rapidly after intravenous administration.

(c) Lipophilic xenobiotics may be carried into tissues by the one-way lymphatic system after intragastrical and intraperitoneal administrations. Such a mechanism could explain irreversibility of the fast distribution processes.

Independently of the exact nature of the fast processes, they are endowed with relative ubiquity and efficiency. Mathematical techniques of modern experimental pharmacokinetics fail to reveal and quantitatively estimate such kind of processes $[1,2]$. As a result, their presence is largely ignored. In the present study, we outlined theoretical considerations and suggested regression and discrimination methods that could form initial steps in working out a formal mathematical apparatus for this area of pharmacokinetics.

\section{References}

[1] S. Björkman, D. R. Wada, B. M. Berling and G. Benoni, Prediction of the disposition of midazolam in surgical patients by a physiologically based pharmacokinetic model, J. Pharmaceutical Sci. 90 (2001), 1226-1240. 
[2] M. Boroujerdi, Pharmacokinetics: Principles and Applications, McGraw-Hill, 2002.

[3] D. W. A. Bourne, Mathematical Modeling of Pharmacokinetic Data, Technomic Publ. Co., Lancaster, PA, 1995.

[4] M. Gibaldi and D. Perrier, Pharmacokinetics, 2nd ed., Dekker, New York/Basel, 1982.

[5] I. Gueorguieva, I. A. Nestorov, S. Murby, S. Gisbert, B. Collins, K. Dickens, J. Duffy, Z. Hussain and M. Rowland, Development of a whole body physiologically based model to characterise the pharmacokinetics of benzodiazepines. 1: Estimation of rat tissue-plasma partition ratios, J. Pharmacokinet. Pharmacodyn. 31 (2004), 269-298.

[6] D. A. Frank-Kamenetskiı̌, Diffusion and Heat Transmission in the Chemical Kinetics, Nauka, Moscow, 1987 (in Russian).

[7] Phenazepam, A. Bogatsky (ed.), Naukova Dumka, 1982 (in Russian).

[8] W. A. Ritschel, Handbook of Basic Pharmacokinetics, Drug Intelligence Publ., 1980.

[9] V. N. Soloviev, A. A. Firsov and V. A. Filov, Pharmacokinetics, Medicine, Moscow, 1980.

[10] D. Varfolomeev and K. G. Gurevich, Biokinetics. Practical Course, Faer-Press, Moscow, 1999 (in Russian).

[11] V. G. Zinkovsky, O. V. Zhuk, N. Ya. Golovenko, S. I. Schukin and A. V. Kolesnikov, Pharmacokinetical subsystem of organisms, in: Pharmacological Analysis of the Receptor-Ligand Interactions, Akademperiodik, 2001, 10-25 (in Ukrainian).

[12] V. G. Zinkovsky and S. I. Schukin, Approach to model-independent method for analysis of disposition of xenobiotics between the blood and tissue under its administration in a single dose, Achievements of Biology and Medicine 2 (2005), 27-32 (in Ukrainian).

[13] V. G. Zinkovsky, O. V. Zhuk and S. K. Sumriy, Pharmacokinetics of a synthetic interferon inducer amixin in mice, Pharmacological Reports 59 (2007), 739-751.

Department of Biotechnology and Molecular Biology

University of Opole

Kominka 4

45-032 Opole, Poland

E-mail: olga_zhuk@uni.opole.pl

theodorr@interia.pl
Department of Biology State University of Odessa Dvoryanska St. 2 Odessa 650000, Ukraine E-mail: lekar.nata@gmail.com

Received on 28.5.2008; 